\title{
Quantum-Chemical Design of Covalent Linkages for Interconnecting Carbon Nanotubes
}

\author{
Fabio Pichierri $^{1}$, Mohammad Khazaei ${ }^{2}$ and Yoshiyuki Kawazoe ${ }^{2}$ \\ ${ }^{1}$ COE Laboratory, Tohoku University, IMRAM, Sendai 980-8577, Japan \\ ${ }^{2}$ Institute for Materials Research, Tohoku University, Sendai 980-8577, Japan
}

\begin{abstract}
The possibility of interconnecting carbon nanotubes (CNTs) through their ends using covalent linkages has been computationally explored. By employing density functional theory (DFT) calculations with Gaussian-type orbitals (GTOs) we have optimized the geometry of four CNTbased model junctions each containing five covalent linkages. The linkages investigated here are the disulfide (-S-S-) and peptide (-CONH-) linkages, which are commonly found in proteins, and the ethylenedioxy $\left(-\mathrm{O}-\mathrm{CH}_{2}-\mathrm{CH}_{2}-\mathrm{O}-\right)$ and ethynyl (-C $\equiv \mathrm{C}-$ ) linkages which can be synthesized in the chemistry laboratory. The geometric and electronic structures computed for these four models are thoroughly discussed. [doi:10.2320/matertrans.N-MRA2007861]
\end{abstract}

(Received October 30, 2006; Accepted May 10, 2007; Published July 11, 2007)

Keywords: carbon nanotubes, covalent linkages, quantum chemistry, materials design

\section{Introduction}

One possible strategy to create novel hybrid nanomaterials with novel and useful properties is that of connecting the building units through covalent linkages. This strategy is somehow inspired by Nature. For example, let us think about the formation of sulfur bridges $(-\mathrm{S}-\mathrm{S}-)$ in proteins which are formed upon oxidation of a pair of thiol groups $(-\mathrm{SH})$ of cysteine amino acid residues that come into close contact upon folding. ${ }^{1)}$ This chemical reaction allows the connection through covalent bonding of two elements of secondary structure, such as a pair of alpha helices, thus providing a more compact tertiary structure of the protein. Another type of covalent linkage that is ubiquitous in proteins is the peptide linkage which derives from the condensation reaction between a carboxylic acid and an amino group.

Following this bio-inspired strategy we have chosen four covalent linkages which allow interconnecting carbon nanotubes (CNTs) through their ends. The title covalent linkages are: a) disulfide linkage, b) peptide linkage, c) ethylenedioxy linkage, and d) ethynyl linkage. The experimental realization of such a proposal requires the functionalization of CNTs with different chemical groups. This is currently an active field of research pursued by many experimental chemists that has already produced interesting results. ${ }^{2,3)}$ For instance, the chemical functionalization of CNTs render them processable in solution (since nonfunctionalized CNTs are insoluble in all organic solvents) as well as facilitates their attachment onto metal surfaces such as that of gold. ${ }^{4)}$ Alternative approaches to interconnect CNTs might exploit either non-covalent interactions, such as hydrogen bonds, or transition metal ions that can act as a nano-scale solder. The former approach has been utilized by Ghadiri and coworkers to prepare peptide nanotubes ${ }^{5)}$ while the latter approach has been recently proposed by one of us (F.P.) in the design of molecular nanotubes made of thiacucurbituril macrocylic units and termed cucurtubes. ${ }^{6}{ }^{6}$ In this study we present the results of molecular orbital (MO) calculations performed within the framework of density functional theory (DFT).

\section{Computational Details}

All the DFT calculations were performed with the exchange-correlation (XC) functional of Perdew, Burke, and Ernzerhof (PBEPBE) $)^{7)}$ in combination with the $3-21 \mathrm{G}$ basis set of Pople and coworkers ${ }^{8)}$ as implemented in the Gaussian 03 software package. ${ }^{9)}$ Geometry optimizations were performed without applying any symmetry constraint while taking full advantage from the automatic density fitting method of Dunlap available only for pure XC functionals. ${ }^{10)}$ The GaussView (version 3.09) ${ }^{11)}$ and Molden (version 4.4) ${ }^{12)}$ graphical user interfaces were employed in all the pre- and post-processing operations. All the DFT calculations were performed on the Linux PC cluster of the COE Laboratory of Computational Chemistry (Tohoku University).

\section{Results and Discussion}

\subsection{Optimized structures}

Figure 1 shows the DFT-optimized structures of the four $\mathrm{CNT}$ junctions (1-4) containing five $-\mathrm{CH}_{2}-\mathrm{S}-\mathrm{S}-\mathrm{CH}_{2}-$ linkages (Fig. 1(a), (b)), five peptide linkages (Fig. 1(c), (d)), five $-\mathrm{O}-\mathrm{CH}_{2}-\mathrm{CH}_{2}-\mathrm{O}-$ linkages (Fig. 1(e), (f)), and five acetylenic $-\mathrm{C} \equiv \mathrm{C}-$ linkages (Fig. $1(\mathrm{~g})$, (h)), respectively. The number of linkages was chosen so as to minimize the conformational flexibility of the model junctions (vide infra) which is expected to increase when less than five chemical bridges are present. Also, by using few linkages (e.g. two) one has to deal with several isomers for each model junction. On the other hand, additional linkages (e.g. from six to ten) cannot be employed due to steric repulsion which has a destabilizing effect on the whole structure. The two CNT parts in each model are modeled with cyclacene units each made of ten fused six-membered carbon rings thus resembling the terminal part of a $(10,0)$ nanotube.

Let us first comment on the optimized structural parameters, namely bond lengths and angles, of these four models. The $\mathrm{S}-\mathrm{S}$ bond lengths in model junction $\mathbf{1}\left(E_{\mathrm{tot}}=\right.$ $-7397.078266 \mathrm{au}$ ) range from 2.274 to $2.290 \AA$. The shortest $\mathrm{S}-\mathrm{S}$ bond is that located at the internal part of the structure 
a)

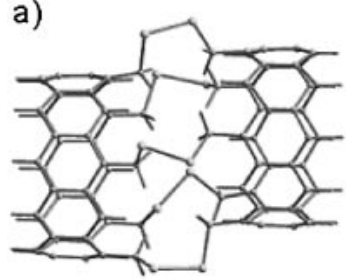

e)

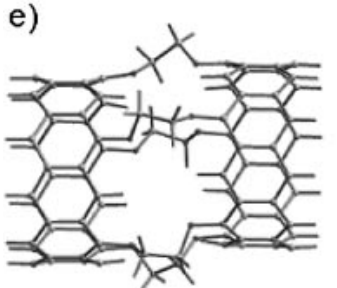

b)

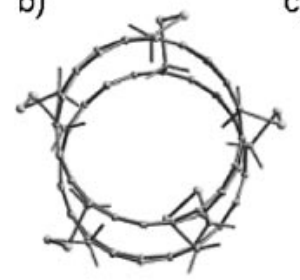

c)

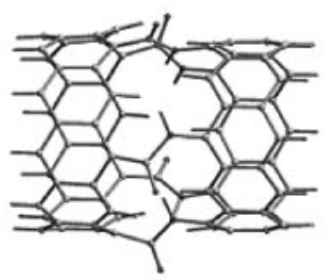

g)

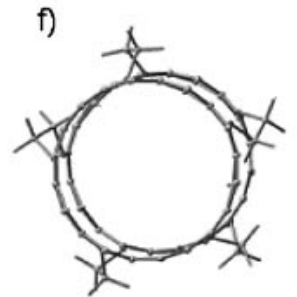

d)

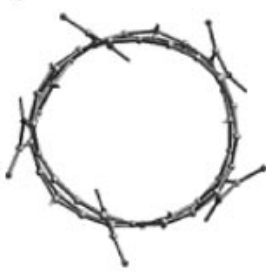

h)

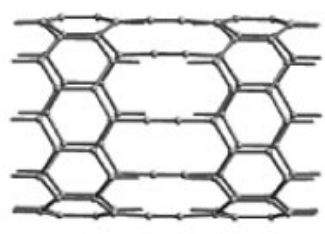

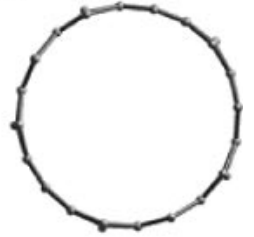

Fig. 1 DFT-optimized structures of four CNT covalent junctions: a,b) disulfide linkage, c,d) peptide linkage, e,f) ethylenedioxy linkage, and $\mathrm{g}, \mathrm{h}$ ) ethynyl linkage.

(see Fig. 1(b)) while the remainder four are found on the exterior of the CNTs. The dihedral angles $\mathrm{C}-\mathrm{S}-\mathrm{S}-\mathrm{C}$ of $\mathbf{1}$ are at $-67,+74,-76,-69$, and +76 degrees. These values indicate that the disulfide linkage can assume a conformation with the dihedral angle at about $\pm 70^{\circ}$. In comparison, the values observed in the crystal structures of proteins are $\pm 90^{\circ} .{ }^{13)}$ The flexibility of this particular model junction which is made of five disulfide linkages, each of them equipped with five rotatable (single) bonds, is responsible for the misalignment of the CNT ends, as shown in Fig. 1(b).

Next we investigated a CNT model junction $2\left(E_{\text {tot }}=\right.$ $-3884.079264 \mathrm{au}$ ) made of five peptide linkages (Fig. 1(c), (d)) which, like the preceding disulfide linkage, are also found in proteins. ${ }^{13)}$ It is worth noticing, however, that the peptide linkage possesses a near flat-like conformation which is due to the partial double-bond character of the $\mathrm{C}-\mathrm{N}$ bond. This is visible from Fig. 1(d) where two peptide linkages are nearly flat. On the other hand, the other three peptide linkages appear to be somewhat concave shaped as a result of their $\mathrm{C}=\mathrm{O}$ and $\mathrm{N}-\mathrm{H}$ bonds being affected by steric repulsions with the nearby $\mathrm{C}-\mathrm{H}$ bonds of the $\mathrm{CNT}$ units. This result indicates that the peptide linkage of $\mathbf{2}$ can be somewhat distorted depending upon its orientation with respect to the CNT units. This structural distortion of the peptide linkage has the effect of significantly increasing the $\mathrm{C}-\mathrm{N}$ bond distance from $\sim 1.39 \AA$ (in the two flat-like linkages) to $\sim 1.45 \AA$ (in the three distorted linkages). It is interesting to notice that deviations from planarity are also observed in the peptide bonds of proteins. ${ }^{14)}$ Another interesting structural effect that we observe in our model junction is the ovalization of one CNT unit whose associated major and minor diameters are at 8.3 and $7.7 \AA$, respectively. Owing to the more rigid structure of the peptide bond with respect to that of the disulfide linkage, the misalignment of the CNT units is minimal (Fig. 1(d)).

The third CNT model junction $3 \quad\left(E_{\text {tot }}=\right.$ $-4183.916733 \mathrm{au})$ that we have investigated here contains five ethylenedioxy linkages $-\mathrm{O}-\mathrm{CH}_{2}-\mathrm{CH}_{2}-\mathrm{O}-$ as shown in Fig. 1(e), (f). This type of linkage is not present inside proteins but it can be synthesized in the chemistry laboratory. The DFT-optimized structure of $\mathbf{3}$ indicates that the average $\mathrm{C}-\mathrm{C}$ bond length is $\sim 1.52 \AA$ while the average $\mathrm{O}-\mathrm{C}$ distance is $\sim 1.49 \AA$. The $\mathrm{O}-\mathrm{C}-\mathrm{C}-\mathrm{O}$ torsion angles are at $+94,+70$, $-59,-69$, and +58 degrees. Hence, these results indicate that this particular linkage is more flexible than the disulfide linkage discussed above. The reason for this difference is that the conformation of the latter is dictated by the stereoelectronic effects involving the lone-pairs of the sulfur atoms whereas in the former such effects are considerably weaker or nearly absent (the lone-pairs on the two oxygen atoms do not interact with each other). Also, we notice that the flexibility of the five $-\mathrm{O}-\mathrm{CH}_{2}-\mathrm{CH}_{2}-\mathrm{O}$ - linkages brings about a certain misalignment of the CNT units, as shown in Fig. 1(f).

The fourth and last CNT model junction $4\left(E_{\mathrm{tot}}=\right.$ $-3424.390996 \mathrm{au}$ ) investigated here contains five ethynyl linkages, as shown in Fig. $1(\mathrm{~g})$, (h). This too is a synthetic (non-natural) fragment which has found useful applications in the syntheses of different functional organic molecules and materials. ${ }^{15)}$ It is worth noticing that the ethynyl unit bears four $\pi$-electrons which together with one $\sigma$-bond contribute to the triple $\mathrm{C} \equiv \mathrm{C}$ bond. The carbon-carbon distances of the five $\mathrm{C} \equiv \mathrm{C}$ bonds in $\mathbf{4}$ are at $1.24 \AA$. Owing to the rigid structure of the ethynyl moiety, the CNT units of $\mathbf{4}$ are very well aligned, as shown on Fig. 1(h).

Before concluding the discussion about the structural properties of the model junctions, it is worth commenting about the relative orientation of the CNT units. As shown in Fig. 2, three deformation modes are expected from the introduction of covalent linkages between CNTs. The first type of deformation concerns the misalignment (or parallel displacement) of the CNT units, which can be quantified by the $d$ parameter. From our DFT results it appears that $d$ ranges from $0 \AA$ in $\mathbf{4}$ to about $2 \sim 3 \AA$ in $\mathbf{1}$ and 3. As expected, $d$ is a function of both the flexibility (related to the number of rotatable bonds) and length of the covalent linkage. The second deformation mode concerns the bending of the CNT units and can be quantified by the bending angle $\alpha$. The DFT-optimized structures of our four CNT junction 


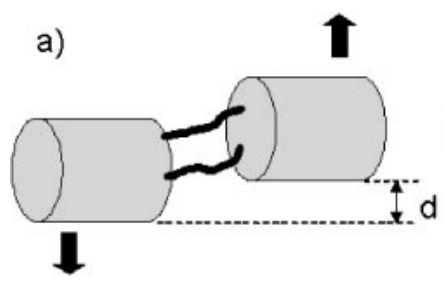

b)

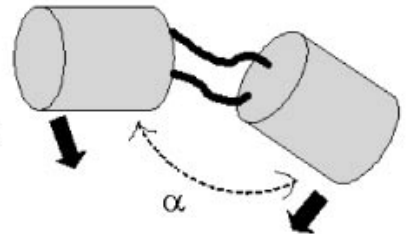

c)

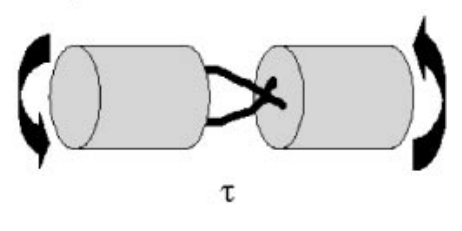

Fig. 2 Deformation modes of the CNT units of 1-4: a) axial misalignment, b) bending deformation, and c) rotation of the CNT units in opposite directions.

a)

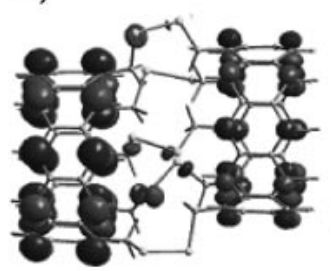

e)

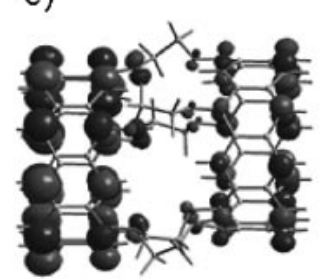

b)

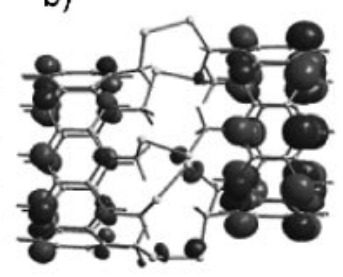

f)

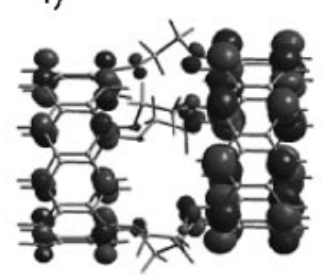

c)

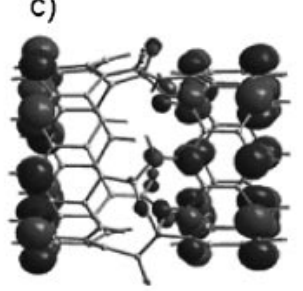

g)

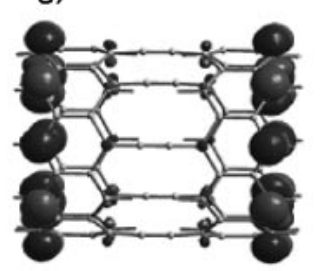

d)

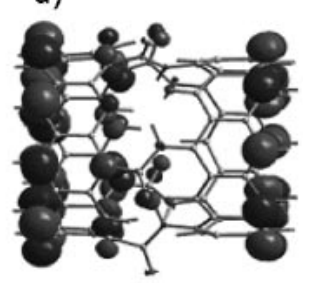

h)

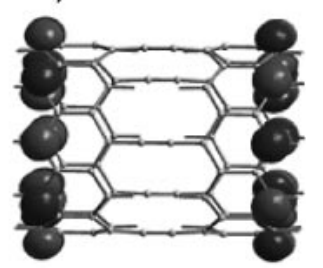

Fig. 3 Frontier molecular orbitals of the four covalent CNT junctions: a,b) disulfide linkage, c,d) peptide linkage, e,f) ethylenedioxy linkage, and g,h) ethynyl linkage. The HOMOs are depicted in a,c,e,f and the LUMOs in b,d,f,h.

models do not show any significant bending effect (see Fig. 1). However, this type of deformation can be expected for CNTs containing either a single covalent linkage or few covalent linkages asymmetrically distributed around the mouth of the nanotubes. The third deformation mode concerns the rotation of the CNT units in opposite directions as quantified by the $\tau$ angle. This kind of deformation is absent in $\mathbf{4}\left(\tau=0^{\circ}\right)$ and very small in $\mathbf{1}$ but it amounts to $5 \sim 10^{\circ}$ in both 2 and 3 . We may expect that either decreasing the number of linkages connecting the CNT units or increasing the lengths of the bridges will enhance the deformation effects observed in these designer junctions.

\subsection{Electronic structure}

One of the interesting features of the electronic structure of finite (molecular) systems is the topology of their frontier orbitals, namely highest-occupied and lowest-unoccupied MOs (HOMO, LUMO). Figure 3 shows the Kohn-Sham frontier orbitals of $\mathbf{1 - 4}$ as computed at the PBEPBE/3-21G/ auto level of theory. As seen from this figure, the topology of the frontier orbitals HOMO and LUMO of $\mathbf{1 - 4}$ is nearly identical inasmuch as it can be reversed simply by rotating the drawings $180^{\circ}$ about the axis perpendicular to the CNT axis.

Differences, however, are observed depending on the nature of the covalent linkage. The CNT junctions $\mathbf{1}$ and $\mathbf{3}$ have similar topologies, with the frontier orbitals semilocalized mainly on one cyclacene unit (left side unit for the HOMO and right side unit for the LUMO). On the other hand, owing to the partial double bond character of the peptide linkages of 2 , the $\mathrm{p}_{\pi}$ orbitals of ten carbon atoms do not contribute significantly to either HOMO (Fig. 3(c)) or LUMO (Fig. 3(d)). In 4, owing to the $\pi$-electron rich ethynyl linkage, the $\mathrm{p}_{\pi}$ orbitals of twenty carbon atoms do contribute to either HOMO (Fig. 3(g)) or LUMO (Fig. 3(h)). The computed HOMO-LUMO energy gaps are as follow: $0.43 \mathrm{ev}$ (1), $0.16 \mathrm{ev}$ (2), $0.46 \mathrm{eV}$ (3), and $0.12 \mathrm{eV}$ (4). The observed trend is dictated by the nature of the covalent linkages and we expect that the same trend will be observed for models connecting larger CNT units.

\subsection{Summary}

We presented the results of DFT calculations performed on four CNT junction models containing different kinds of covalent linkages connecting the CNT units. The calculations indicate that the junctions made of disulfide (1) and ethylendioxy (3) linkages are quite flexible whereas that with the ethynyl linkage (4) yields a rigid structure. The model junction containing five peptide linkages (2) possesses an intermediate degree of structural flexibility due to the partial double-bond character of the peptide bond. Hence, selecting the proper covalent linkage allows one to control the flexibility of the CNT junction. Also, given the presence or absence of $\pi$-electrons on the bridge allows one to tune the electronic properties of the resulting CNT junction thereby suppressing either totally or partially the electronic communication between the CNT units. These results are expected to contribute to advancements in the fields of hybrid nano- 
materials and nanoelectronics. We are currently extending this work by using plane-wave DFT calculations with and without periodic boundary conditions (PBCs) so as to investigate the properties of larger CNT junction models.

\section{Acknowledgments}

F.P. thanks the 21st century COE project "Giant Molecules and Complex Systems" of Tohoku University for financial support. M.K. thanks the Institute for Materials Research for hospitality and financial support.

\section{REFERENCES}

1) J. M. Berg, J. L. Tymoczko and L. Stryer: Biochemistry, 6th edition (W. H. Freeman, New York, 2006).

2) D. Tasis, N. Tagmatarchis, A. Bianco and M. Prato: Chem. Rev. 106 (2006) 1105-1136.

3) S. Banerjee, T. Hemraj-Benny and S. S. Wong: Adv. Mater. 17 (2005)
$17-29$

4) J. Liu et al:: Science 280 (1998) 1253-1256.

5) J. D. Hartgerink, J. R. Granja, R. A. Milligan and M. R. Ghadiri: J. Am. Chem. Soc. 118 (1996) 43-50.

6) F. Pichierri: Chem. Phys. Lett. 403 (2005) 252-256.

7) J. P. Perdew, K. Burke and M. Ernzerhof: Phys. Rev. Lett. 77 (1996) 3865-3868.

8) J. S. Binkley, J. A. Pople and W. J. Hehre: J. Am. Chem. Soc. 102 (1980) 939-947.

9) Gaussian 03, Revision C.02, M. J. Frisch et al., Gaussian, Inc., Wallingford CT, 2004

10) B. I. Dunlap: J. Mol. Struct. (Theochem) 529 (2000) 37-40.

11) GaussView, Version 3.09, Roy Dennington II, Todd Keith, John Millam, Ken Eppinnett, W. Lee Hovell and Ray Gilliland, Semichem, Inc., Shawnee Mission, KS, 2003.

12) G. Schaftenaar and J. H. Noordik: J. Comput.-Aided Mol. Design 14 (2000) 123-134.

13) J. S. Richardson: Adv. Protein Chem. 34 (1981) 167-339.

14) M. W. MacArthur and J. M. Thornton: J. Mol. Biol. 264 (1996) 11801195.

15) F. Diederich, P. Stang and R. R. Tykwinski: Acetylene Chemistry: Chemistry, Biology, and Material Science (John Wiley \& Sons, New York, 2005). 